\title{
FATIGUE CRACK GROWTH IN PATCHED PLATES UNDER CONSTANT AMPLITUDE AND FLIGHT SIMULATION LOADINGS
}

\author{
R. Chandra* and R. Sunder*** \\ * Structures Divisions NAL. Bangalore, India \\ **Materials Divisions NAL, Bangalore, India
}

ABSTRACT

Fatigue crack propagation was studied in aluminium alloy plates reinforced by adhesive bonding a unidirectional carbon fibre epoxy patch across the crack path. The tests were carried out under constant amplitude as well as flight simulation combat aircraft spectrum loading. Comparative tests were also conducted on unpatched plates. The composite patch considerably reduces crack growth rates. The improvement in resistance to fatigue crack growth is more pronounced under spectrum loading conditions.

\section{KEY WORDS}

Fatigue crack growth; patched plates; constant amplitude loading; flight simulation loading.

\section{INTRODUCTION}

The 'Fail Safe' design concept is widely used in the design of aircraft structural components. The design of such 'damage tolerant' structures is governed by detailed specifications(N.N.,1974,1977), which in principle ensure that a detectable fatigue crack will not grow to critical proportions in the period between routine inspections. Fatigue cracked components are often repaired in service. Standard repair schemes normally involve strengthening the component by connecting a member by means of bolts or rivets. This practice involves the risk of undesirable stress concentration at attachment points.

Recent technological advcances in fibre reinforced composite materials and adhesive bonding have led to the development of 
efficient repair schemes in which (i) the load is transferred from the component to the reinforcement without serious stress concentration, (ii) the properties of the reinforcement can be tailored to suit particular application and (iii) the composite repair patch does not contribute significantly to increase in weight of the component.Boron-epoxy, boron-aluminium and carbon -epoxy have been used in some repair schemes of aircraft structures (Baker, 1976, 1978).

This investigation was carried out to evaluate the fatigue crack growth resistance of L-72 aluminium alloy plates reinforced with carbon fibre epoxy patch. The next section of the paper describes the experimental procedures used in the study. This is followed by a discussion of obtained results and their implications for practical application of carbon fibre patches to repair cracked panels in service.

\section{EXPERIMENTAL PROCEDURES}

\section{Test Specimens}

Single Edge Notch (SEN) specimens cut along the rolling direction from 2 mm thick L-72 Al-Cu-Zn alloy were used in the tests. The specimen width was $75 \mathrm{~mm}$. A $7 \mathrm{~m}$ deep, 0.5 mo wide slit was milled using a slitting saw to serve as the crack initiator. The specimens were divided into two batches. One batch of specimens was reinforced with a composite patch. It may be noted that the reinforcement was made on one side only. Such a specimen is schematically described in Fig.l. The other batch was used to generate baseline data to evaluate fatigue performance of the patched specimens. The following procedure was used to prepare the patched specimens.

Unidirectioal carbon-epoxy composite plates of 1 mm thickness were made by filament winding technique using a flat plate mandrel. High modulus carbon fibre (Grafil E/HM-S) from Courtaulds Ltd, England and compatible epoxy were used to make carbon-epoxy composite plate. The patch strips of 100 x $15 \mathrm{~mm}$ were cut by using diamond. cutter equipment.

Film adhesive Redux 312 L from CIBA-GEIGY of UR was used for bonding the carbon-epoxy composite patch to notched specimen. This adhesive was selected in view of its superior shear strength. Aluminium alloy specimens were pretreated as recommended by the manufacturer of the adhesive. This process involves degreasing and chemical treating as per DTD $\mathbf{9 1 5 8 ( i i )}$ of U.K. specifications. The curing schedule for the adhesive is: $120+5$ deg.C for 30 minutes, . Pressure = $350 \mathrm{RPa}$. 
The Fatigue Crack Propagation (FCP) tests were carried out on a $250 \mathrm{RN}$, computer controlled Instron servo hydraulic testing machine. Hydraulic grips were used and hence bending due to neutral axis shift was constrained.

The tests were carried out under constant amplitude as well as flight simulation loadings. To enable subsequent comparison of results both types of specimens were tested runder identical stress levels. The constant amplitude tests were carried out at four different stress ranges with zero stress ratio. The flight simulation loading tests were also carried out at four different mean (1g) stress levels. Details of stress levels used in the tests appear in Table 1. It may be noted that the stress values for the patched specimens do not account for the slightly increased cross section due to the composite patch.

The flight simulation load sequence was generated by a real time computer program. The sequence is based on a load spectrum, derived for a combat aircraft (Sunder and Raju, 1983). The spectrum is stored in computer memory in the form of a joint probability matrix of peak-trough occurences: The matrix was truncated to contain load levels in the range of og to $6 \mathrm{~g}$ with a step of $0.5 \mathrm{~g}$ (a total of 13 levels). Each flight of loads consists of pseudo-random peak-trough excursions from this matrix - 35 load cycles per flight. A typical segment of this loading appears in Fig. 2.

Crack propagation tests on the unpatched specimens were carried out in the automatic mode. In this mode, the computer monitors crack length through periodic measurements of unloading compliance. The hardware and software used for these measurements is described by sunder(1984). A table of crack length versus number of load cycles is maintained by the computer. The step of this table is $0.5 \mathrm{~mm}$ of crack extension. At the conclusion of the test, this table along with pertinent test details are recorded into a disc file.

The patched specimens were tested in the manual mode. In this mode, the progress of the fatigue crack was visually monitored with a magnifying glass. Readings of crack length versus number of load cycles were registered every 1 mm upto a crack length of $20 \mathrm{~mm}$ and every $2 \mathrm{~mm}$ thereafter. Test results in the form of FCP lives from $9 \mathrm{~mm}$ to failure are summarised in Table 1.

The test data were processed by a computer program which uses an incremental polynominal to approximate the crack growth curve. The derivative of this function defines crack growth rate. The program also includes graphic routines to plot the results of analysis. 
The baseline constant amplitude crack growth rates obtained at different stress levels are plotted versus stress intensity factor range $\langle\Delta \nabla$ ) in Fig.3. The crack growth rates during these tests always exceeded $0.0001 \mathrm{~mm} /$ cycle. These data appear to cover the ranges of shear mode and rapid fatigue crack propagation.

Figures 4-7 show the effect of the patch on crack growth rates under constant amplitude loading at four different stress levels. The following observations can be made with reference to the effect of a patch on FCP under constant amplitude (CA) loading: 1. The crack growth rates in the patched specimens as compared to the unpatched ones progressively decrease with crack length. The reduction is even more noticeable after the crack completely traverses the section under the patch.2. The reduction in crack growth rates is more pronounced under higher load levels.

Figures 9-12 show the effect of the patch on crack growth rates under flight simulation (FS) loading. These data are essentially similar to those obtained under constant amplitude loading. However it follows from the crack growth rate curves as well as from the table of crack propagation lives that: 1. The beneficial effect of the composite patch is more pronounced under FS loading conditions.2. Mean stress under FS loading influences FCP life in patched specimens even more strongly than stress range under $C A$ loading. This is obvious from the curves in Fig.13 which show this effect on life ratio $\mathrm{Np} / \mathrm{Nunp}$ (the ratio of number of cycles/flights at failure for patched specimens to those for unpatched specimens). From the curves in Fig.13a,we find that for constant amplitude loading conditions, a 100 percent increase in stress range(from 50 to $100 \mathrm{MPa}$ ) results in the ratio $\mathrm{Np} / \mathrm{Nunp} 3$ to 7 . In contrast, a similar extent of increase in mean stress under FS loading (see Fig.13b), i.e., from 15 to $30 \mathrm{MPa}$ results in an increase of this ratio from 2.3 to 141

In an attempt to generalise these results, the test data were processed to inpirically derive the apparent stress intensity factor from baseline material data for CA and FS loading conditions which appear in Figs. 3 and 8. It was assumed that the similarity concept is valid for a crack in a patched plate, i.e.. for similar stress intensity factor range, one should expect similar crack growth rates, irrespective of whether the crack is growing in a patched or unpatched material. The results of this analysis are presented as apparent patch factor versus crack length, where apparent patch factor, $\mathrm{P}$, represents the ratio of SIF (stress intensity factor) for a crack in an unpatched plate to that in a patched plate. 
One would expect a unique relationship between SIF and crack length for a patched plate. The results in Fig.14 however point to an apparent influence of stress level as well as load sequence on the empirically determined patch factors.

At first glance the results in Fig.14 appear to contradict those in Fig.13, inspite of the fact that both were obtained from the same set of experimental data. While the results in Fig.13 indicate a greater beneficial effect of the patch under FS loading conditions, particularly at higher mean stress levels, the curves in Fig.14 show that the apparent patch factor for FS loading condition at any given crack length is as a rule lower than that under CA condition.

This anomaly is easily explained if one considers the baseline data in Figs.3 8. Assuming a log linear relationship between crack growth rate and stress intensity factor one gets:

$$
P=\left[\frac{(d a / d N)_{u n p}}{(d a / d N)_{p}}\right]^{1 / m}
$$

where, $(\mathrm{da} / \mathrm{dN})_{\text {unp }}$ is crack growth rate for unpatched case

$$
(\mathrm{da} / \mathrm{dN})_{p} \text { is crack growth rate for patched case at }
$$

$m$ is the slope of the baseline (da/dN) versus $\mathrm{AK}$ data (in log scale).

This equation would be valid for both CA as well as FS loading conditions. The baseline CA crack growth rate data in Fig. 3 indicate a slope $\mathrm{m}=3.328$ for $\mathrm{da} / \mathrm{dN}$ values below 0.01 $\mathrm{mm} /$ cycle. For FS loading conditions one gets $\mathrm{m}=7.5$ from the data in Fig.8. It follows that for similar reduction in stress intensity factor the corresponding reduction in crack growth rate is much more pronounced under FS loading conditions,

It is beyond the scope of this paper to study the reasons for the observed differences in the behaviour of a fatigue crack under CA and FS loading conditions. One may tentatively suggest the following explanation for the observed anomalies.

Due to load interaction effects a fatigue crack shows accelerated growth under load cycles of higher magnitude and retarded growth under the smaller load cycles (Schijve,1975). Obviously it is these larger load cycles which control the FCP process. With increase in mean stress level, the effective stress ranges of the larger load cycle fall into the region of crack growth rates exceeding $0.01 \mathrm{~mm} / \mathrm{cycle}$ (see Fig.3). The slope $\mathrm{m}$ in this region rapidly increases due to the proximity of the critical stress intensity factor. Even at relatively low mean stress levels, a large proportion of crack growth under FS loading occurs at rates exceeding $0.01 \mathrm{~mm} / \mathrm{cycle}$, (i.e., greater values of $\mathrm{m}$ ). Therefore, the effectiveness 
of the patch is more pronounced under FS loading conditions.

\section{CONCLUSION}

1. FCP tests were carried out on patched and unpatched aluminium alloy plates under constant amplitude and flight simulation loading conditions. 2. The results of this study indicate that careful consideration should be given to service load sequences and overall mean stresses while designing and evaluating composite repair patches.

\section{REFERENCES}

Baker,A.A., and M.M.Hutchinson (1976). Tech.Memo. ARL/Mat 366 , Aero.Res. Lab. (Melbourne).

Baker,A.A (1978). Composites, 9(1), 11-16.

N.N (I974). MIL-Spec. 83444, USAF.

NN (1977). FAA,14 FCR Part 25, Par. 25.571.

Sunder,R., and $\mathbb{R} . \mathbb{N} \cdot$ Raju (1983). Proc. SEECO 83, 256-278.

Sunder, $R$ (1984). Proc.Sisth Int.Conf.Fracture (New Delhi), Accepted for presentation.

Schijve,J(1975). Report VTH-193, Delft University.

\section{ACKNOWLEDGEMENT}

Financial support of this work was provided by Aeronautics Research and Development Board. Authors wish to thank Mr.D.T.Srinivasa and Mr.M.N.Neelakante Gowda for their help in conducting the tests.Thanks are due to $\mathrm{Dr}$. K.N.Raju for valuable suggestions during the course of this work. Thanks are due to Mr.R.Purushothaman for his help in preparing the manuscript.

\section{TABLE 1 Summary of Tests}

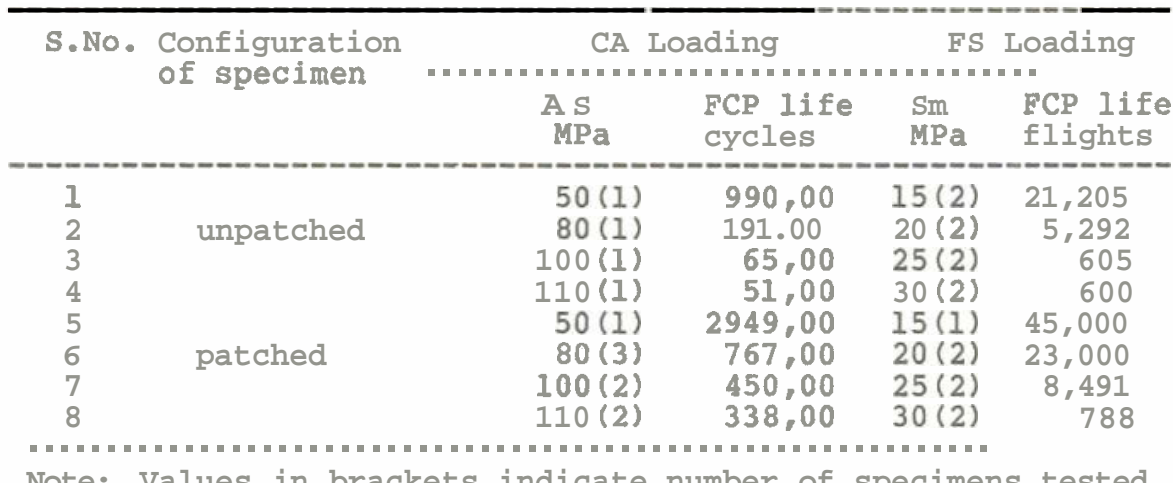

Note: Values in brackets indicate number of specimens tested. 


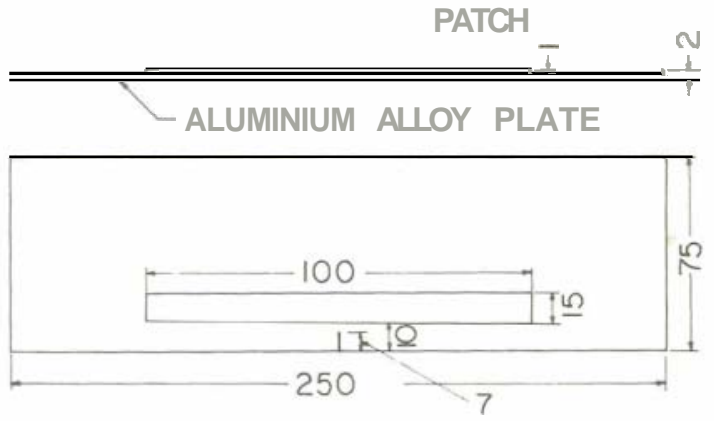

Fig.1. Geometry of specimen

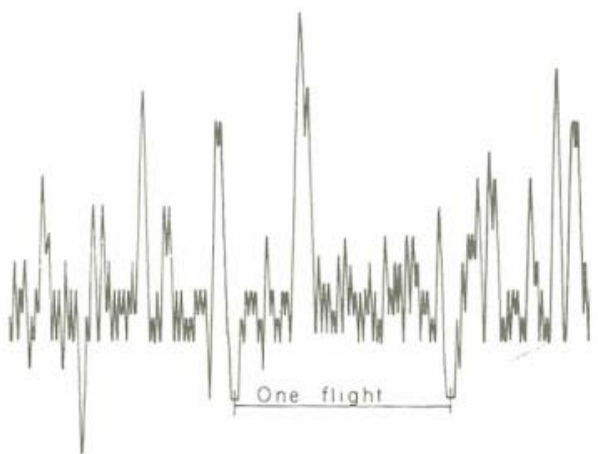

Fig.2. Typical load sequence

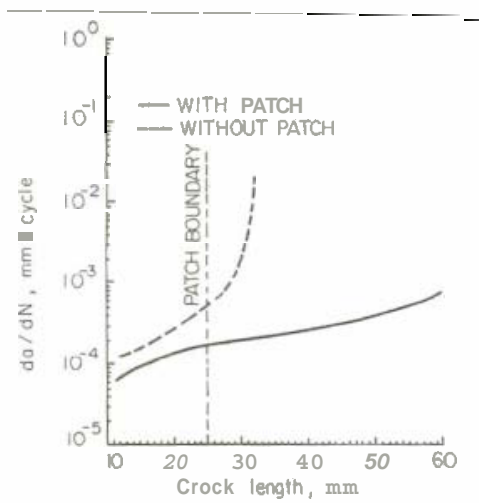

Fig.4. da/dN vs a, $\triangle S=50 M P a$

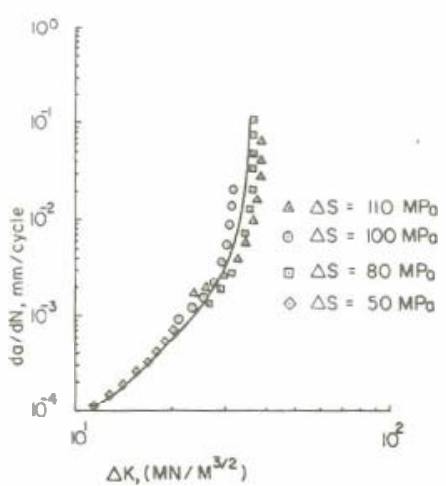

Fig.3.da/dN- $\triangle R$ for $C A$ loading

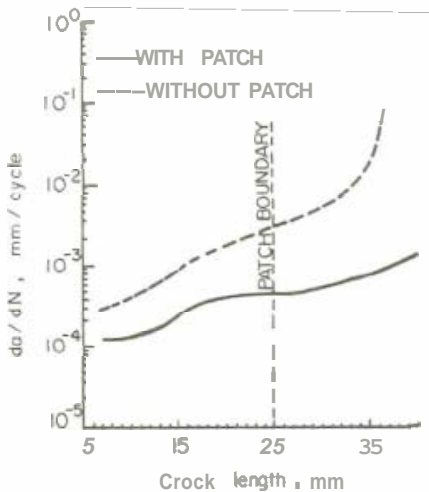

Fig. $5 \mathrm{da} / \mathrm{dN}$ vs a, $\triangle \mathrm{S}=80 \mathrm{MPa}$ 


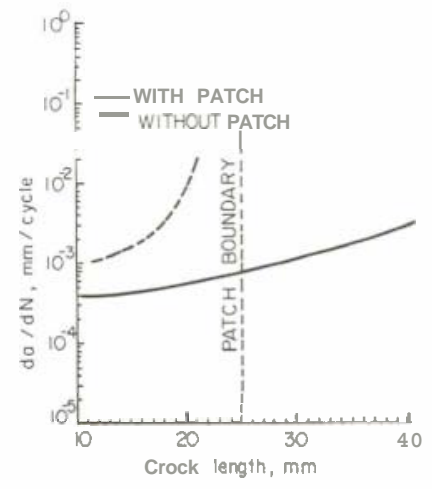

Fig.6. $\mathrm{da} / \mathrm{dN}$ vs a, $\Delta s=100 \mathrm{MPa}$

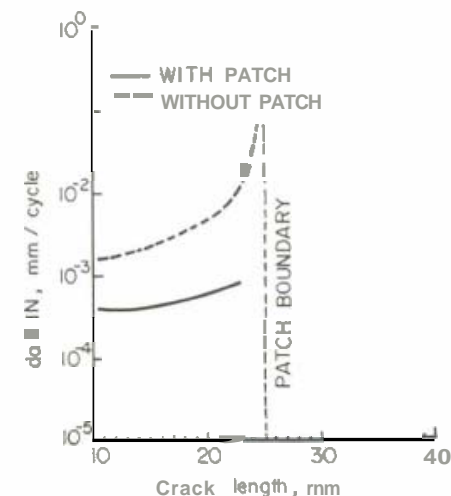

Fig. $7 \mathrm{da} / \mathrm{dN}$ vs $a, \Delta S=110 \mathrm{MPa}$

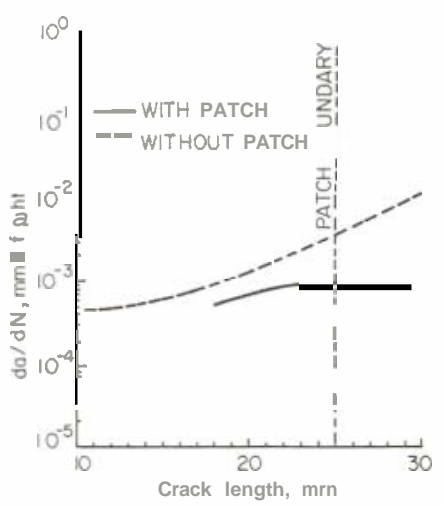

Fig.9. da/dN vs a, $S m=15 \mathrm{MPa}$

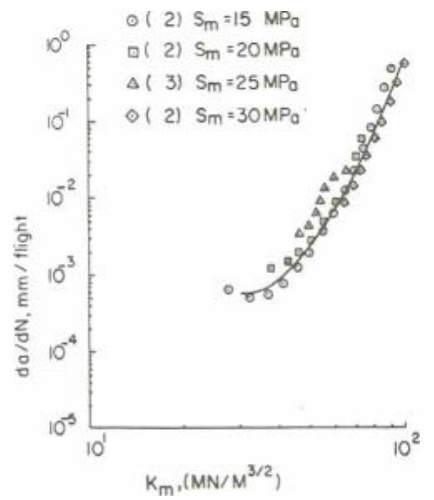

Fig.8. da/dN-Rm for FS loading

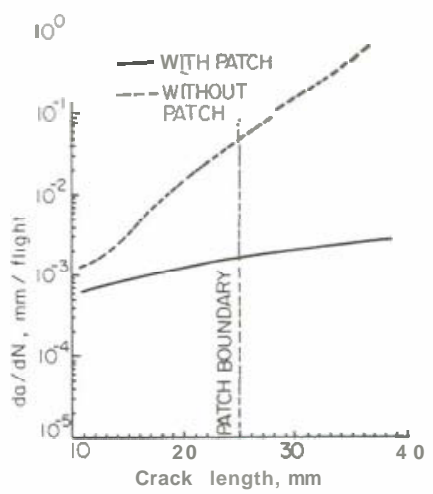

Fig.10, da/dN vs $a, S m=20 \mathrm{MPa}$ 

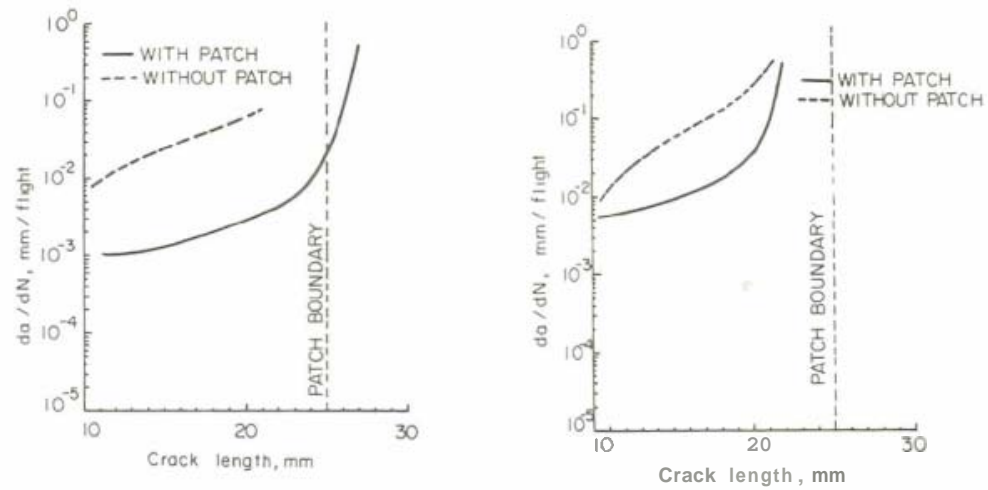

Fig.11.da/dN vs a, Sm=25MPa Fig.12. da/dN vs a, Sm=30MPa

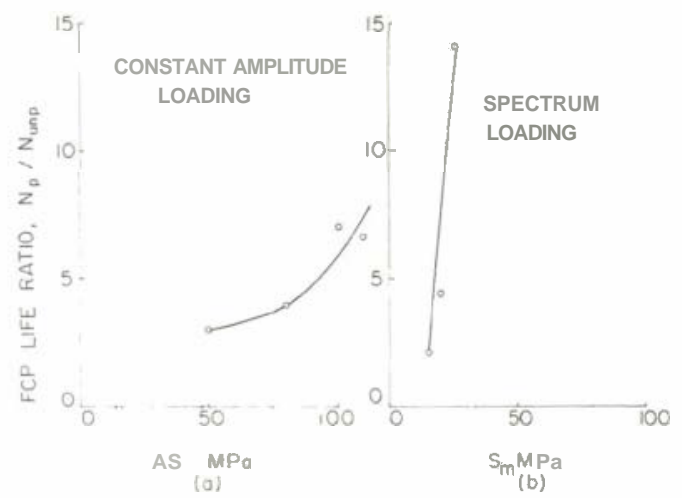

Fig. 13. FCP life enhancement

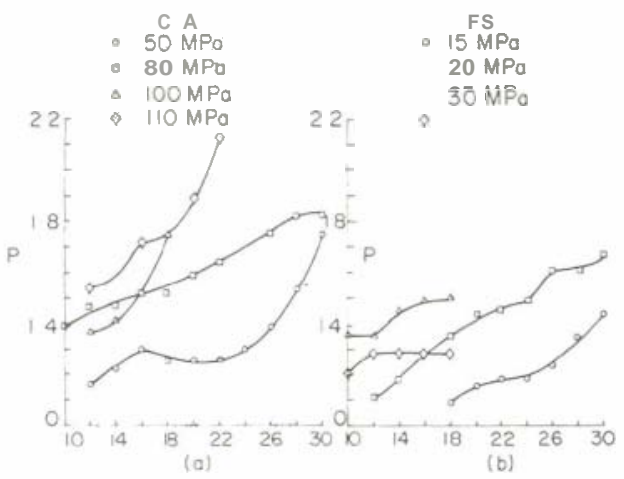

Fig.14. Apparent patch factors 